\title{
NODAL signaling components regulate essential events in the establishment of pregnancy
}

\author{
Craig B Park ${ }^{1,2}$ and Daniel Dufort ${ }^{1,2,3}$ \\ ${ }^{1}$ Division of Experimental Medicine, ${ }^{2}$ Centre for the Study of Reproduction at McGill and ${ }^{3}$ Department of Obstetrics \\ and Gynecology, McGill University Health Centre, Royal Victoria Hospital, 687 Pine Avenue West, Room F3-24, \\ Montreal, Quebec, Canada H3A 1A1
}

Correspondence should be addressed to D Dufort at Department of Obstetrics and Gynecology, McGill University Health Centre, Royal Victoria Hospital; Email: daniel.dufort@mcgill.ca

\begin{abstract}
Successful mammalian reproduction is dependent on a receptive and nurturing uterine environment. In order to establish pregnancy in humans, the uterus must i) be adequately prepared to receive the blastocyst, ii) engage in a coordinated molecular dialog with the embryo to facilitate implantation, and iii) undergo endometrial decidualization. Although numerous factors have been implicated in these essential processes, the precise network of molecular interactions that govern receptivity, embryo implantation, and decidualization remain unclear. NODAL, a morphogen in the transforming growth factor $\beta$ superfamily, is well known for its critical functions during embryogenesis; however, recent studies have demonstrated an emerging role for NODAL signaling during early mammalian reproduction. Here, we review the established data and a recent wave of new studies implicating NODAL signaling components in uterine cycling, embryo implantation, and endometrial decidualization in humans and mice.

Reproduction (2013) 145 R55-R64
\end{abstract}

\section{Introduction}

Mammalian reproduction requires a healthy, protective, and nurturing maternal environment throughout gestation. In order to establish pregnancy in humans, the uterus must first be adequately prepared to receive the embryo in the event of successful copulation and fertilization. Under the primary control of the ovarian steroid hormones, estrogen and progesterone, the outer endometrial layer undergoes significant proliferative expansion and the uterus enters a state of receptivity during which implantation is possible (Wang \& Dey 2006). Once uterine receptivity is attained, complex reciprocal signaling between the uterus and embryo must then render the blastocyst competent to endometrial attachment and penetration. As a result, the available 'window of implantation', when the uterus is receptive and the blastocyst is competent, requires highly regulated and synchronous development of both the embryo and the endometrial tissue (Carson et al. 2000, Wang \& Dey 2006). Following the initiation of implantation, the underlying uterine stroma must undergo decidualization to provide an initial source of nourishment and to mediate the expansion and invasion of the embedded extraembryonic trophectoderm (Ramathal et al. 2010).
Infertility is a common clinical issue worldwide and is defined as the inability of a couple to conceive naturally after 24 months of trying. Infertility affects $7-10 \%$ of couples and is attributable to a number of factors including ovarian dysfunction, hormonal or genetic disorders, reproductive tract pathologies, male gamete insufficiencies, or even environmental factors (WHO Technical Report Series 1992). It is currently believed that the majority of early pregnancy loss arises from defects that occur immediately before or during the process of implantation, such as improper uterine conditioning (receptivity) or miscues in uterine-embryo molecular interactions (Wang \& Dey 2006). Infertility due to defective uterine receptivity or repeated implantation failure is not easily circumvented by assisted reproductive technologies. Strikingly, after all known causes of infertility have been clinically investigated and ruled out, $\sim 15-30 \%$ of cases remain idiopathic (termed 'unexplained infertility') (Quaas \& Dokras 2008).

Although the major physiological events that govern early human reproduction have been characterized, the molecular pathways that mediate receptivity, implantation, and decidualization remain relatively unclear (Dey et al. 2004). As a result, overcoming complications associated with repeated implantation 
failure has been difficult. This issue is compounded by the limited ability to conduct in vivo research on uterine-embryo interactions in humans. Nonetheless, identifying the molecular mechanisms that facilitate uterine cycling/receptivity, embryo implantation, and decidualization in various animal models has provided significant information toward understanding human implantation. In this review, we explore both the established data and a recent wave of new studies implicating the components of the NODAL signaling pathway in mediating the necessary events for embryo implantation and the establishment of pregnancy in both humans and mice (summarized in Table 1). Furthermore, we discuss future research and necessary experimentation that could aid in the translation of this knowledge from the laboratory to the clinic.

\section{NODAL signaling}

NODAL, a morphogen in the transforming growth factor $\beta$ (TGFB) superfamily, was first identified by transgenic screening in the mouse that produced a recessive lethal mutation (termed 413.d) that severely affected gastrulation beginning on day 7.5 post-coitum (Conlon et al. 1991, lannaccone et al. 1992). Targeted mutation of a novel TGFB-like gene, Nodal, which mapped alongside 413.d, recapitulated the hyperplasia of the embryonic and extraembryonic ectoderm, leading to failed mesoderm induction and embryonic lethality by day 10.5 (Zhou et al. 1993, Conlon et al. 1994, Jones et al. 1995). Together, these studies underscored the essential role of NODAL during mesendoderm induction and introduced an intriguing new member of the TGFB superfamily. Since its discovery, NODAL has been

Table 1 Components of the NODAL signaling pathway implicated in early reproduction.

\begin{tabular}{|c|c|c|c|c|}
\hline Process & Species & Component & Observation(s) & Reference(s) \\
\hline \multirow[t]{10}{*}{$\begin{array}{l}\text { Uterine cycling } \\
\text { and receptivity }\end{array}$} & \multirow[t]{8}{*}{ Human } & \multirow[t]{2}{*}{ NODAL } & $\begin{array}{l}\text { NODAL mRNA detected during the proliferative and early-secretory } \\
\text { phases. Protein in stroma, epithelial layers, and lumen }\end{array}$ & Papageorgiou et al. (2009) \\
\hline & & & $\begin{array}{l}\text { NODAL expression observed in endometrial samples of healthy } \\
\text { patients. No difference in proliferative and secretory phases }\end{array}$ & Torres et al. (2009) \\
\hline & & \multirow[t]{3}{*}{ LEFTYA (EBAF) } & $\begin{array}{l}\text { LEFTYA mRNA is high during secretory and menses } \\
\text { (opposite NODAL). Protein in glandular epithelium } \\
\text { and surrounding stroma }\end{array}$ & $\begin{array}{l}\text { Tabibzadeh et al. }(1997,1998) \\
\text { and Papageorgiou et al. } \\
\text { (2009) }\end{array}$ \\
\hline & & & $\begin{array}{l}\text { No decrease in } L E F T Y \text { expression in patients with abnormal uterine } \\
\text { bleeding; inexplicably high in unexplained infertility }\end{array}$ & $\begin{array}{l}\text { Kothapalli et al. (1997) and } \\
\text { Tabibzadeh et al. (2000) }\end{array}$ \\
\hline & & & $\begin{array}{l}\text { LEFTYA is a local mediator of endometrial breakdown and repair by } \\
\text { triggering matrix metalloproteinase (MMP) expression }\end{array}$ & Cornet et al. $(2002,2005)$ \\
\hline & & \multirow[t]{2}{*}{ TDGF1/CRIPTO } & $\begin{array}{l}\text { TDGF1/CRIPTO mRNA consistently detected throughout the menstrual } \\
\text { cycle; higher during secretory phases. Protein mirrors NODAL }\end{array}$ & Papageorgiou et al. (2009) \\
\hline & & & $\begin{array}{l}\text { TDGF1/CRIPTO expression observed in endometrial samples of } \\
\text { healthy women. No difference in proliferative and secretory phases }\end{array}$ & Torres et al. (2009) \\
\hline & & $\begin{array}{l}\text { SMAD2/3, } \\
\text { SMAD4 }\end{array}$ & $\begin{array}{l}\text { Human endometrium expresses the SMAD transducers; however, } \\
\text { induction is shown through TGFBs (not discussed) }\end{array}$ & Luo et al. (2003) \\
\hline & \multirow[t]{2}{*}{ Mouse } & LEFTY1 & $\begin{array}{l}\text { Lefty } 1 \text { expressed throughout the cycle (lowest at estrus) and pregnancy; } \\
\text { mirrored by MMP. Protein in glands/lumen/stroma }\end{array}$ & Tang et al. (2005a) \\
\hline & & $\begin{array}{l}\text { SMAD2, } \\
\text { SMAD4 }\end{array}$ & $\begin{array}{l}\text { Smad } 2 \text { and } S \text { mad } 4 \text { mRNA found in glandular and luminal epithelium. } \\
\text { Smad } 4 \text { consistent; Smad } 2 \text { higher at di/proestrus }\end{array}$ & Liu et al. (2004) \\
\hline \multirow[t]{7}{*}{$\begin{array}{l}\text { Embryo } \\
\text { implantation }\end{array}$} & \multirow[t]{7}{*}{ Mouse } & \multirow[t]{2}{*}{$\begin{array}{l}\text { NODAL } \\
\text { (embryonic) }\end{array}$} & $\begin{array}{l}\text { Null and hypomorphic Nodal mice exhibit defects during } \\
\text { embryogenesis and placentation; implantation unaffected }\end{array}$ & $\begin{array}{l}\text { lannaccone et al. (1992) } \\
\text { and Ma et al. (2001) }\end{array}$ \\
\hline & & & $\begin{array}{l}\text { Nodal/Bmp5 double mutant embryos display decidua crowding with } \\
\text { heterozygous and wild-type embryos }\end{array}$ & Pfendler et al. (2000) \\
\hline & & \multirow[t]{2}{*}{$\begin{array}{l}\text { NODAL } \\
\text { (uterine) }\end{array}$} & $\begin{array}{l}\text { Nodal expression generates a banding pattern along the uterine horn at } \\
\text { implantation; regulated by embryonic factors }\end{array}$ & Park \& Dufort (2011a) \\
\hline & & & $\begin{array}{l}\text { Uterine Nodal knockout females demonstrate subfertility due to } \\
\text { reduced rates of establishing pregnancy }\end{array}$ & Park et al. (2012) \\
\hline & & LEFTY1/2 & $\begin{array}{l}\text { In vivo gene transfer of Lefty overexpression into the uterus leads to } \\
\text { implantation failure }\end{array}$ & Tang et al. (2005b) \\
\hline & & FURIN, PACE4 & $\begin{array}{l}\text { Uterine-derived proprotein convertases are able to cleave and activate } \\
\text { embryonic-derived NODAL at the time of implantation }\end{array}$ & Mesnard \& Constam (2010) \\
\hline & & SPC6 & $\begin{array}{l}\text { The LEFTY processing enzyme, SPC6, is expressed at the implantation } \\
\text { site during embryo attachment and penetration }\end{array}$ & Rancourt \& Rancourt (1997) \\
\hline \multirow[t]{5}{*}{$\begin{array}{l}\text { Decidualiza- } \\
\quad \text { tion }\end{array}$} & \multirow[t]{3}{*}{ Human } & NODAL & $\begin{array}{l}\text { NODAL protein not observed in mid- to late-secretory endometrium } \\
\text { (including epithelial layers); not detected in HESC }\end{array}$ & Stoikos et al. (2008) \\
\hline & & LEFTYA/B & $\begin{array}{l}\text { Microarray and northern blot analysis demonstrate } \\
\text { that } L E F T Y A \text { and } L E F T Y B \text { are highly induced } \\
\text { in decidua that support implantation }\end{array}$ & $\begin{array}{l}\text { Brar et al. (2001) and } \\
\text { Tabibzadeh \& } \\
\text { Hemmati-Brivanlou (2006) }\end{array}$ \\
\hline & & SPC5/6 & $\begin{array}{l}\text { HESC decidualization in vitro leads to increased SPC5/6. Blocking } \\
\text { SPC } 5 / 6 \text { cleaving ability reduced decidualization }\end{array}$ & Okada et al. (2005) \\
\hline & \multirow[t]{2}{*}{ Mouse } & SPC $5 / 6$ & $\begin{array}{l}\text { Ratio of mature/precursor LEFTY is increased at time and location of } \\
\text { implantation; independent of embryo control }\end{array}$ & Tang et al. (2005c) \\
\hline & & LEFTY1/2 & $\begin{array}{l}\text { Comprehensive in vitro and in vivo analysis demonstrates that LEFTY } \\
\text { mediates decidualization by autocrine regulation }\end{array}$ & Tang et al. (2010) \\
\hline
\end{tabular}


identified as a key regulator in several additional processes during embryogenesis, including left-right axis specification and anterior-posterior patterning (Brennan et al. 2002, Takaoka et al. 2006).

NODAL is first translated as a precursor proprotein that is posttranslationally modified before exocytosis (Blanchet et al. 2008). The proprotein must then be proteolytically cleaved to its mature form at the R-X-X-R consensus sequence by the subtilisin-like proprotein convertase (SPC) 1 or SPC4 (also known as FURIN and PACE4) in order to effectively bind and activate the signaling pathway (Beck et al. 2002). Mature NODAL ligand binds to an extracellular membrane-bound receptor complex comprising type I (ACVR1B or ACVR1C) and type II (ACVR2A or ACVR2) activin-like receptors in addition to a glycosylphosphatidylinositollinked EGF-CFC co-receptor (TDGF1/CRIPTO or CFC1/ CRYPTIC) (Gritsman et al. 1999, Reissmann et al. 2001). The EGF-CFC co-receptor is essential for NODAL signaling by recruiting the convertases to the receptor complex site and facilitating NODAL binding to the ACVR1B/ACVR1C type I receptor (Blanchet et al. 2008). Upon receptor complex formation, ACVR2A/ACVR2 phosphorylates ACVR1B/ACVR1C, which in turn phosphorylates the intracellular signal transducers SMAD2 or SMAD3 (Kumar et al. 2001). SMAD2/3 then interacts with SMAD4 and additional transcription factors in the nucleus (e.g. FOXH1, MIXER, and p53) to facilitate DNA binding and regulate target gene expression (Ross \& Hill 2008). NODAL signaling is regulated at numerous levels throughout the cascade including, but not limited to, posttranslational glycosylation, proteolytic cleavage, extracellular antagonism, miRNA interference, receptor degradation/recycling, and transducer ubiquitination (for detailed reviews of NODAL signaling, see Schier (2003, 2009)).
As a morphogen, NODAL is able to diffuse over a long range within a tissue and acts directly on distant cells in a concentration-dependent manner (Chen \& Schier 2001). Numerous downstream targets of NODAL signaling have been identified; however, target genes vary considerably by species, tissue, and function. Interestingly, the most common result of NODAL signaling is the production of more NODAL ligand and its diffusible inhibitor, LEFTY, which restricts signaling by binding to both the EGF-CFC co-receptor and NODAL directly (Meno et al. 1999, Chen \& Shen 2004). NODAL signaling induces self-propagation exclusively through a conserved FOXH1-dependent intronic enhancer (asymmetric enhancer (ASE)) while LEFTY production is controlled through a similar ASE and additional enhancer elements (Hamada et al. 2002, Norris et al. 2002). As a result, NODAL and LEFTY generate complex patterns of positive and negative regulation that are believed to constitute a reaction-diffusion mechanism (Box 1; Muller et al. 2012). LEFTY proteins are divergent members of the TGFB superfamily that comprise LEFTYA and LEFTYB in humans and their homologs LEFTY2 and LEFTY1 in mice (Schier 2003). LEFTY homology is based on the distribution of enhancer elements of the genes, but there is no tendency for LEFTYA protein to be more related to LEFTY2 than LEFTY1. In fact, the LEFTY pairs within a species have a much greater sequence identity than across species (Fig. 1; Kosaki et al. 1999). In this review, the precise LEFTY examined is indicated wherever possible; however, several early studies conducted before the identification of the second LEFTY gene do not make this distinction feasible and are simply denoted as 'LEFTY'.

In addition to the critical functions during embryo development, several studies have uncovered potential roles for NODAL signaling throughout mammalian

Box 1 NODAL and LEFTY as a potential reaction-diffusion mechanism The classic reaction-diffusion mechanism postulates that a locally expressed 'activator' can generate self-organizing patterns of activity by inducing both its own expression (positive feedback) and that of a potent 'inhibitor' (negative feedback). In order to create these spatiotemporal patterns, often manifested in the form of radial stripes (a target pattern), banding, or dots, both components must possess diffusing properties with the inhibitor exhibiting a longer range (paraphrased, 'local activation - long-range inhibition') (Turing 1990). Interestingly, small initial differences in activator-inhibitor concentration along a plane can quickly become amplified due to the self-catalytic nature of activator production and long-range restriction (Kondo 2002). NODAL and LEFTY demonstrate the required properties to constitute a reaction-diffusion pair as NODAL signaling has been shown to induce both NODAL self-production (activator) and LEFTYexpression (inhibitor) (Chen \& Schier 2002). Furthermore, both NODAL and LEFTY were demonstrated to diffuse over long distances in a chick model, with LEFTY displaying a faster rate (Sakuma et al. 2002). Although self-organization of NODAL and LEFTY in vivo has not been verified empirically, it is believed that these fundamental characteristics play a role in left-right axis specification and embryonic patterning (Schier 2009). In addition to the pattern formation derived from the reaction-diffusion model, NODAL signaling adds another layer of complexity due to its morphogenic properties, which permit differing target gene expression as concentration diminishes from the source. 


\begin{tabular}{c|cccc|c}
\multicolumn{1}{c}{} & LEFTYA & LEFTYB & LEFTY1 & LEFTY2 \\
\cline { 2 - 5 } LEFTY2 & $81 \%$ & $82 \%$ & $90 \%$ & - \\
LEFTY1 & $81 \%$ & $82 \%$ & - & \\
LEFTYB & $96 \%$ & - & $\begin{array}{c}\text { LEFTYA/EBAF (human) } \\
\text { LEFTY2 (mouse) }\end{array}$ & $\begin{array}{l}\text { LEFTYB (human) } \\
\text { LEFTY1 (mouse) }\end{array}$ \\
\hline LEFTYA & - & & \multicolumn{2}{c}{}
\end{tabular}

Figure 1 Inter- and intra-species amino acid sequence identity of homologous LEFTY proteins.

reproduction. NODAL is believed to act as a mediator during the follicular atresia of non-dominant follicles before ovulation (Wang et al. 2006, Wang \& Tsang 2007) and NODAL also appears to play a complex role during trophoblast invasion and placentation (Nadeem et al. 2011, Park \& Dufort 2011b). However, the precise mechanics underlying these functions are not fully understood. Accumulating data regarding NODAL and LEFTY in the uterus, combined with their unique interactive properties, has also made NODAL signaling an interesting candidate in facilitating embryo implantation and ensuring the successful establishment of pregnancy.

\section{NODAL signaling components implicated in uterine preparation, cycling, and receptivity}

\section{Human}

In order for successful implantation to occur, the endometrium must first be morphologically prepared to receive the free-floating blastocyst into the uterine wall. In humans, this is achieved by sequential phases of significant endometrial proliferation and secretion that correlate temporally with ovulation and potentially with embryo implantation. During the mid-secretory phase, between 7 and 10 days following ovulation, the uterus becomes receptive and is able to support implantation (Wang \& Dey 2006). If pregnancy is not established, efficient breakdown (menses) and regeneration of the outermost endometrial layer, the functionalis, is absolutely critical to prepare the uterus for the next cycle of proliferation, ovulation, and potential conception.

Using uterine samples obtained from healthy nonpregnant women, it was recently demonstrated that NODAL and LEFTYA exhibit distinctly opposite patterns of expression that alternate throughout the menstrual cycle (Papageorgiou et al. 2009). NODAL mRNA was first detected by RT-PCR at the early-proliferative phase and steadily increased during the late-proliferative and early-secretory stages (48-fold) before decreasing substantially from the mid-secretory phase into menses. Interestingly, LEFTYA mRNA displayed a nearly opposite pattern of expression. Minimal LEFTYA expression was observed throughout the proliferative and early/ mid-secretory phase before increasing dramatically (155-fold) during the late-secretory and menses stages
(Papageorgiou et al. 2009). These opposite and alternating patterns of expression over the course of the menstrual cycle suggest that NODAL and LEFTYA play important phase-dependent roles in endometrial preparation, breakdown, and/or tissue repair. Furthermore, one such transition in NODAL and LEFTYA levels occurs during the mid-secretory phase when uterine receptivity is acquired.

At the histological level, NODAL protein was detectable throughout the stroma, luminal epithelium, and glandular epithelial cells by immunolocalization. Although the level of NODAL in the luminal and glandular epithelium remained consistent and detectable at all phases of the menstrual cycle, the stromal intensity declined significantly during the late-secretory and menstrual phases (Papageorgiou et al. 2009). As a result, NODAL staining in the large stroma compartment followed the same cyclical pattern observed when NODAL mRNA was quantified by RT-PCR, suggesting that the stroma cells account for the majority of endometrial NODAL expression. Interestingly, NODAL proprotein was also detected in uterine fluid, indicating that NODAL is secreted into the lumen of the uterus that contains the free-floating blastocyst (Papageorgiou et al. 2009). Unfortunately, measuring whether NODAL proprotein levels in uterine fluid fluctuate at the precise time of embryo implantation is not feasible in humans due to technical limitations.

In addition to the recent characterization of NODAL localization in the uterus, LEFTYA, originally denoted 'endometrial bleeding-associated factor' (EBAF), has previously been implicated in uterine physiology. As the initial name suggests, $L E F T Y A / E B A F$ was first identified in the endometrium as a novel TGFB superfamily member that exhibited increased expression during the late-secretory and menses phases as the uterine lining undergoes tissue breakdown and shedding (Tabibzadeh et al. 1997). LEFTYA immunoreactivity was restricted to the glandular epithelium, adjacent stromal cells, and the endothelial cells lining the uterine spiral arterioles (Tabibzadeh et al. 1998). More recently, the mechanisms underlying LEFTYA-mediated endometrial breakdown following ovarian steroid withdrawal have been uncovered. In addition to LEFTYA expression increasing at the perimenstrual phase in vivo, a marked increase was also observed in ex vivo proliferative endometrial explants that were cultured without ovarian hormone supplementation to simulate menses conditions (Cornet et al. 2002). Increased LEFTYA expression was subsequently followed by increased matrix metalloproteinase 1 (MMP1/interstitial collagenase), MMP3 (stromelysin 1), and MMP9 (gelatinase B) expression, which are enzymes that proteolytically degrade the uterine functionalis. Furthermore, recombinant LEFTYA stimulated the production of MMP3, MMP7 (matrilysin 1), and MMP9 in proliferative explants; however, these observations were prevented by ovarian 
steroid hormone supplementation that mimics the proliferative phase of the endometrium (i.e. a nonmenses state) (Cornet et al. 2002, 2005). Therefore, these studies demonstrate that LEFTYA acts as a local, uterine mediator of endometrial breakdown following the reduction in ovarian hormones (Tabibzadeh 2005).

From a clinical standpoint, LEFTY has been implicated in cases of abnormal uterine bleeding in addition to idiopathic infertility in human patients. Consistent with its proposed roles during menstruation, LEFTY expression was found throughout the endometrium during all stages of the menstrual cycle in women who experienced irregular or excessive shedding (menorrhagia) (Kothapalli et al. 1997). LEFTY protein was detected in the endometrial fluid of fertile women (much like NODAL) and is also secreted by transfected endometrial cells in culture. Secreted LEFTY was reduced during uterine receptivity in fertile subjects; however, abundant LEFTY was inexplicably detected during the receptive phase in several infertile females (Tabibzadeh et al. 2000). Together, these results indicate that $L E F T Y$ misexpression during receptivity may underlie irregular uterine bleeding and some causes of idiopathic infertility.

In order for the NODAL signaling pathway to be activated in the uterus, the essential receptors and co-receptors must be present. The EGF-CFC co-receptor CRIPTO/TDGF1 (Papageorgiou et al. 2009) and activin receptors, ACVR1B, ACVR2A, and ACVR2B (Jones et al. 2002), have been detected in the uterus at all stages of the cycle with moderately higher levels of expression observed during the secretory phase. CRIPTO/TDGF1 immunolocalization mirrored that of the NODAL ligand; however, additional CRIPTO/TDGF1 protein was detectable around the uterine spiral arterioles (Papageorgiou et al. 2009). Finally, Torres et al. (2009) also observed NODAL, CRIPTO/TDGF1, and ACVR2A mRNA in the uterus of healthy women; however, a significant difference in NODAL expression was not observed between the denoted proliferative and secretory categories. Once such explanation for the discrepancy would be the limited number of categories applied in this study (two) as NODAL mRNA, when evaluated using six distinct phases, is detectable in both the proliferative (early, mid, and late) and secretory (early) phases. Nonetheless, NODAL and its associated receptors, co-receptor, and inhibitor are expressed in the endometrium and exhibit dynamic patterns of expression during the menstrual cycle.

\section{Mice}

In mice, the estrus cycle is characterized by phases of moderate uterine growth and expansion (proestrus, estrus) followed by stages of endometrial degeneration and quiescence (metestrus, diestrus respectively) (Wood et al. 2007). Estrus culminates with ovulation and, if accompanied by copulation, fertilization occurs in the oviduct on day 0.5 post-coitum. During early reproduction (days 0.5-4.0), the uterus is pre-receptive to implantation and the lumen is less hostile to the blastocyst than the nonpregnant state. On the morning of day 4, a surge of estrogen renders the uterus receptive to implantation until day 5.5, when the opportunity for pregnancy has passed and uterine environment becomes unfavorable to embryo survival (refractory period) (Wang \& Dey 2006).

Recently, Nodal expression in the uterus was documented throughout the estrus cycle and pregnancy using immunohistochemistry and a transgenic NodallacZ mouse strain that reports the sites of Nodal transcription (Park \& Dufort 2011a). In this study, neither Nodal expression nor localized protein was detected at any point in the estrus cycle of nonpregnant mice. However, Nodal was highly expressed in the uterine glandular epithelium throughout the pre-receptive phase (days 0.5-3.5) and positive immunoreactivity was detected along the luminal surface, suggesting NODAL secretion (Park \& Dufort 2011a). Alternatively, Lefty 1 mRNA was previously documented in uterine samples isolated from nonpregnant mice at all stages of the estrus cycle with moderately lower levels of expression during the estrus phase (stage of uterine expansion) (Tang et al. 2005a). In the event of copulation, uterine Lefty 1 expression increased gradually throughout the pre-receptive phase before reaching its highest point at the time of implantation. LEFTY1 protein was observed in the basal lamina, uterine glands (including lumen secretions), stroma compartment, and vessel walls. Similar to the observations in human tissue, the levels of MMP expression mirrored those of Lefty1, leading to high levels of collagen content during estrus (when Lefty/MMP is reduced) followed by low collagen quantities during diestrus (Tang et al. 2005a). This pattern of expression suggests that LEFTY may play a conserved role in ensuring healthy uterine cycling by regulating $M M P$ expression and endometrial turnover in mammals.

The intracellular SMAD proteins are essential transducers of the TGFB superfamily, including the NODAL signaling pathway. Importantly, Smad2 and Smad4 were recently characterized throughout estrus and early pregnancy in the mouse (Liu et al. 2004). Both factors were observed in the glandular and luminal epithelium by in situ hybridization, with Smad2 showing slightly higher expression during diestrus and proestrus whereas Smad4 demonstrated similar intensity throughout the cycle. Furthermore, during early pregnancy, Smad2 and Smad4 signal was abundant in the epithelial layers and displayed a steady increase in relative intensity as pregnancy progressed (days 0.5-4.5) (Liu et al. 2004). Therefore, although the precise function(s) of NODAL signaling during the peri-implantation period requires additional investigation, the essential components of the 
cascade are co-expressed in the endometrium (mainly glandular or luminal epithelium), indicating that pathway activation is feasible.

\section{Nodal signaling components linked to implantation and embryo spacing}

\section{Mice}

Upon entering the uterine lumen, the free-floating blastocysts align within the uterine horn to ensure adequate spacing between conceptus sites. The receptive uterus and embryo then undergo complex reciprocal signaling, which is mediated by locally produced growth factors, cytokines, lipid mediators, adhesion molecules, and transcription factors (Dey et al. 2004). The physical process of embryo implantation is divided into three phases. During i) apposition, stromal edema causes the uterine lumen to close and interdigitate, thereby allowing the blastocyst to contact the luminal epithelium. Next, during ii) attachment, a stable adhesion of the trophectoderm to the uterine lining is achieved by an assortment of adhesion molecules (e.g. integrins, cadherins, and mucin 1), which prevent the embryo from dislodging. Finally, blastocyst attachment initiates apoptosis of the luminal epithelia and allows the embryo to iii) penetrate into the underlying stroma (Wang \& Dey 2006). Although numerous factors have been implicated in these processes, a comprehensive understanding of the molecular events that facilitate implantation remains to be elucidated.

As previously introduced, null Nodal embryos die on day 10.5. These results suggest that NODAL derived from the embryonic genome is itself not essential for implantation. Interestingly, however, embryonic NODAL and bone morphogenic protein 5 (BMP5) exhibit a potential compensatory interaction during implantation and embryo spacing. Nodal and Bmp5 double knockout embryo were generated and examined on day 7.5 of gestation. Strikingly, deciduas that contained a Nodal ${ }^{-1-}: B m p 5^{-1-}$ double knockout embryo often contained two to four additional embryos of differing genotypes with separate
Reichert's membranes (Pfendler et al. 2000). Although Nodal ${ }^{-1-}: B m p 5^{-1-}$ embryos were phenotypically mutant, the additional embryos in the same deciduum were morphologically normal. Therefore, the authors of this study suggest that signals emanating from the implanting embryo to indicate that an implantation crypt has been occupied are disrupted (Pfendler et al. 2000). As such, Nodal and Bmp5 double mutation reveals a potential role for these factors during the implantation reaction and, based on the decidua crowding, appear to facilitate adequate embryo spacing within the uterus.

Implantation is governed by both embryonic and uterine factors. Uterine Nodal expression and protein localization at the time of implantation were recently documented in mice. Interestingly, whole-mount Nodal expression produced a banding pattern along the proximal-distal axis of the uterine horn that correlated exclusively with the inter-implantation nodes (Fig. 2; Park \& Dufort 2011a). This maternal Nodal expression is directed by the blastocyst as pseudopregnant females lost Nodal expression by day 4.5 ; however, the pattern was restored by wild-type embryo transfer experiments (Park \& Dufort 2011a). Therefore, Nodal expression is influenced by the uterine-embryo molecular cross talk at the time of implantation. Considering i) the embryonic Nodal $^{-/-}: B m p 5^{-/-}$knockout, ii) the banding pattern of uterine Nodal expression directed by the embryo, and iii) the self-regulating nature of NODAL signaling, it is tempting to hypothesize that embryonic and maternal derived NODAL functions together to facilitate implantation and ensure adequate embryo spacing.

Lefty 1 expression is highest in the mouse endometrium at the time of implantation (Tang et al. 2005a). Similar to the LEFTYA overexpression observed in human patients with unexplained infertility, in vivo gene transfer of exogenous LEFTY led to significant implantation failure in mice. Using either a Lefty retroviral expression vector system or a liposome-mediated introduction of an expression vector, Tang et al. (2005b) observed implantation rates of $\sim 47.6$ and $47.8 \%$ relative to the control vectors. As these methods used to overexpress Lefty encompassed the entire uterine horn, the site-specific (a)

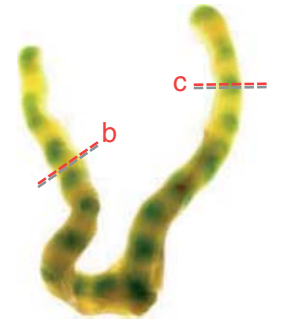

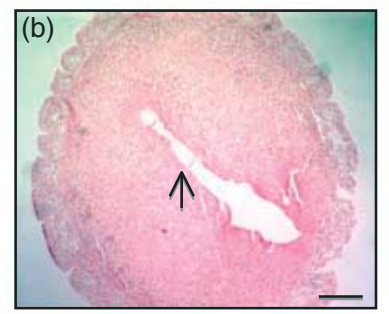

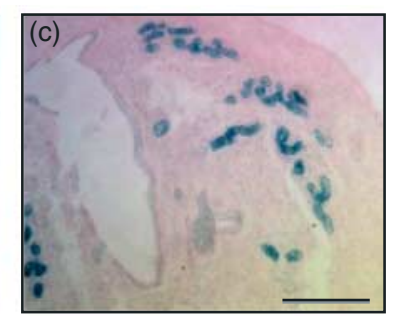

Figure 2 Nodal expression in the uterus at the time of embryo implantation. (a) Whole-mount Nodal-lacZ uterus isolated on day 4.5 post-coitum displaying a distinctive banding pattern along the proximal-distal axis of the uterine horn when stained for $\beta$-galactosidase activity. (b) Transverse histological section of a non-stained band (demonstrated in (a)) depicting glandular epithelium devoid of positive Nodal-lacZ signal and an implanting blastocyst (arrow). (c) Transverse section through a positively staining band (illustrated in (a)) demonstrating robust glandular epithelial expression in the inter-implantation space. Sections were counterstained with Nuclear Fast Red (Sigma); Scale bars indicate $200 \mu \mathrm{m}$. 
expression of Nodal between implantation sites would undoubtedly be disrupted in addition to any other Lefty 1 targets. Moreover, a maternal reproductive tract-specific Nodal knockout strain was recently introduced (Park et al. 2012). In addition to exhibiting a severe placental phenotype, uterine Nodal knockout females displayed significant subfertility during early reproduction. Although the early phenotype was not described, pregnancy failure manifests before day 5.5 and may arise from defective implantation and/or endometrial decidualization. Taken together, eliminating Nodal from a maternal source, or increasing Lefty expression in the uterine lumen, adversely affects the ability to establish pregnancy. Although the potential function of these factors during embryo implantation could be independent, it is an intriguing correlation given the intricacies of the NODAL signaling pathway.

A novel biosensor of proteolysis recently revealed new information regarding the in vivo activity of the convertases FURIN and PACE4. Using the 'cell surfacelinked indicator of proteolysis' (CLIP) system, it was demonstrated that FURIN and PACE4 are active in the embryo immediately before and during the process of implantation (Mesnard \& Constam 2010). Despite this, FURIN and PACE4 double knockout embryos still reported CLIP cleavage, embryonic NODAL processing, and downstream expression of NODAL target genes during early embryogenesis. In light of these results, the authors conclude that maternal proprotein convertases are active in the uterus during implantation and capable of cleaving embryonic NODAL (Mesnard \& Constam 2010). In addition to NODAL processing, SPC6 has also been observed at the implantation site and implicated in the molecular events that occur during, or immediately following, embryo penetration (Rancourt \& Rancourt 1997). It is currently believed that SPC5/6 are the only convertases capable of processing LEFTY, thereby suggesting that NODAL and LEFTY are both cleaved and activated at the intimate maternal-fetal interface during implantation. Although the precise mechanics of NODAL and LEFTY processing require further investigation, the CLIP system and SPC6 activity highlight an intriguing interaction between maternal and embryonic components of the NODAL signaling pathway during implantation.

\section{NODAL signaling components and endometrial decidualization}

Decidualization is the physiological process by which endometrial stromal cells, under the control of progesterone and/or cAMP, differentiate into large, rounded decidual cells that support embryo growth and maintain early pregnancy (Ramathal et al. 2010). Unlike rodents, which only undergo decidualization at the implantation site during blastocyst attachment, human decidualization is initiated with each menstrual cycle in preparation for potential copulation. Beginning at the late-secretory phase, stromal cells surrounding the spiral arteries first differentiate into decidual cells and undergo morphological, biochemical, and vascular changes that are absolutely essential to facilitate embryo implantation. Successful blastocyst attachment and penetration results in the spread of decidualization, encompassing a larger portion of the uterine endometrium as the pregnancy proceeds (Ramathal et al. 2010).

\section{Human}

As previously described, NODAL expression in the stromal compartment of the human endometrium is highest during proliferation before declining substantially during the secretory stage. NODAL protein was, however, still detectable around the luminal and glandular epithelium at a steady state throughout all phases of the menstrual cycle (Papageorgiou et al. 2009). In the context of decidualization, an earlier study by the same investigators explored the roles of numerous TGFB superfamily members during decidua formation (Stoikos et al. 2008). Intriguingly, NODAL protein was not observed in mid- to late-secretory endometrial samples as would be reported in Papageorgiou et al. (2009). However, these studies did use different anti-NODAL antibodies and the epithelial immunoreactivity is relatively low compared with the stromal intensity observed during the proliferative phase. Correlating with the mRNA data, perhaps NODAL is highly expressed and secreted from the glandular epithelium during the proliferative phases after which NODAL protein remains variably detectable during the secretory stage depending on protein stability or antibody sensitivity. NODAL was also undetected in human endometrial stromal cells (HESC) in vitro when examined in both the non-decidualized and decidualized state. These results support the aforementioned data as the HESC were derived primarily from secretory phase uterine biopsies (day 10-21) and contain 97\% pure stromal cells (Stoikos et al. 2008). The absence of NODAL expression and immunoreactivity during decidualization is especially interesting considering the striking decline of NODAL mRNA during the secretory phase in humans (which decidualize in each cycle) and the banding pattern that shows decreased Nodal expression at the implantation site in mice (which only decidualize locally in the presence of the implanting blastocyst).

Alternatively, LEFTY is believed to be a critical mediator during the differentiation of human endometrial cells (Tabibzadeh \& Hemmati-Brivanlou 2006). LEFTYA and LEFTYB were demonstrated by microarray analysis and verified by northern blot to be one of the most abundantly induced factors in the decidua that supports implantation (Brar et al. 2001). Moreover, 
expression of the LEFTY processing enzyme SPC5/6 was significantly increased within HESC during decidualization in vitro (Okada et al. 2005). Intense SPC5/6 immunoreactivity was noted in the cytoplasm of the decidualized cells compared with non-decidualized cells, and, interestingly, functional attenuation of SPC5 cleaving ability by antisense morpholinos reduced decidualization (Okada et al. 2005). These cumulative results indicate that LEFTY is both present and able to be converted to its biologically active form within the endometrium as it undergoes decidualization. Removing the ability of SPC5 impairs decidualization, demonstrating a key role in facilitating the differentiation of human stromal cells.

\section{Mice}

Despite fundamental differences in the timing and initiation of decidualization in humans and mice, LEFTY processing by SPC $5 / 6$ is an essential and conserved element of the mouse decidual response. During the peri-implantation period, both precursor and processed LEFTY appear in equal quantities in the uterus; however, significantly more processed LEFTY protein was evident by days 3-5 post-coitum. Interestingly, as early pregnancy is established, the ratio of processed/ precursor LEFTY protein is significantly higher at the decidualizing implantation site relative to the interimplantation node (Tang et al. 2005c). Increased Spc5/6 expression and LEFTY processing was also observed during artificial decidualization with oil or uterine trauma, indicating that proteolytic cleavage of LEFTY is involved in decidualization but independent of embryonic control. The direct processing of LEFTY during decidualization has been demonstrated in vitro with cells derived from human uterine fibroblasts (HuF) and endometrial stroma (SHT290); however, processing and secretion appeared variable depending on the decidualization stimulus and cell type investigated (Tang et al. 2005c).

Recently, in vitro and in vivo experimentation uncovered additional details regarding the function of LEFTY during decidualization. The addition of recombinant LEFTY to HuF cells during decidualization reduced the secretion of decidual markers (IGFBP1, PRL) and decreased the expression of transcription factors critical to decidualization (ETS1 and FOXO1). Conversely, Lefty knockdown during decidualization increased decidual marker secretion and essential transcription factor levels (Tang et al. 2010). These results were supported in vivo, as mouse uteri injected with retroviral particles that transduce LEFTY had no indication of decidualization on day 9. Furthermore, overexpression of Lefty using a doxycycline-induced Tet-On system increased Lefty in the epithelial layers of the uterus (sevenfold) and markedly reduced fertility as gauged by litter size. Doxycycline-induced Lefty overexpression also decreased artificial decidualization ability. Based on these cumulative results, the authors of this study propose an elegant model in which endogenous LEFTY acts as a molecular switch during decidualization. Under the control of decidualization cues, LEFTY is released from decidua cells and functions in an autocrine manner to both inhibit differentiation and maintain the decidualized state (Tang et al. 2010).

\section{NODAL and early reproduction: future directions}

In this review, we have compiled and revisited numerous studies that have implicated components of NODAL signaling in early reproduction. Although these studies present compelling evidence that NODAL signaling is essential for the establishment of pregnancy, substantial research is still required to elucidate and verify the underlying molecular mechanisms involved. Notably, all investigated components of NODAL signaling were observed (and often co-expressed) in the uterine endometrium indicating that pathway activation is possible; however, the definitive downstream cellular and genetic targets have not yet been explored. Furthermore, many members of the NODAL signaling cascade are shared among other TGFB pathways. Where does NODAL fit into the larger network of interactions that govern uterine/menstrual cycling, embryo implantation, and decidualization? Why is Nodal initially expressed at the implantation site and what factors emanating from the murine blastocyst restrict Nodal during uterine-embryo molecular cross talk?

As we have seen, independently eliminating NODAL or overexpressing LEFTY in the uterus severely impacts fertility in mice. Based on the uterine banding pattern, it may be necessary to revisit these experiments and investigate how modulating NODAL or LEFTY affects the quantity and spatiotemporal pattern of the opposing factor. The nature of NODAL-LEFTY interplay along the uterine horn is a potentially intriguing system that remains to be thoroughly examined given the potential of an in vivo reaction-diffusion mechanism (Muller et al. 2012). It would be interesting to examine whether implantation drives the self-organization of NODAL and LEFTY into opposite and alternating levels of high and low concentration along the horn. Aside from these properties of organization, it is tempting to speculate that NODAL and LEFTY work toward ensuring either adequate embryo spacing and/or restricting the spread of decidualization in humans and mice.

The cumulative observations detailed here are especially interesting given the recent confirmation of uterine NODAL expression in humans and documented cases of aberrant LEFTY expression in patients with unexplained infertility. In order to translate the accumulating knowledge from the laboratory to the clinic, significant experimentation is required. Is NODAL also affected in uterine samples obtained from cases of 
idiopathic infertility in which LEFTY levels are elevated? Are NODAL and LEFTY interactions perturbed in women who experience repeated implantation failure despite clinical intervention and fertility treatment? NODAL was found to be a secreted factor and constituent of uterine luminal fluid in both mice and humans. What is the role of secreted NODAL, and can supplementing this factor counteract dysregulated LEFTY expression in uterine tissue? As the data accumulates, it is increasingly more evident that NODAL signaling components play critical roles during mammalian reproduction and the establishment of pregnancy. However, it is also apparent that a greater understanding of the molecular interplay between NODAL and LEFTY during these reproductive events is necessary to apply this knowledge toward improving reproductive health and, ultimately, combating human infertility.

\section{Declaration of interest}

The authors declare that there is no conflict of interest that could be perceived as prejudicing the impartiality of the research reported.

\section{Funding}

This work was supported by grant MOP-82780 from the Canadian Institutes of Health Research to D Dufort.

\section{Acknowledgements}

The authors thank Drs Hugh Clarke, Riaz Farookhi and Makoto Nagano for critical reading of the manuscript. C B Park is supported by awards from the MUHC-RI and CIHR and is a member of the CIHR-REDIH program.

\section{References}

Beck S, Le Good JA, Guzman M, Ben Haim N, Roy K, Beermann F \& Constam DB 2002 Extraembryonic proteases regulate Nodal signalling during gastrulation. Nature Cell Biology 4 981-985. (doi:10.1038/ ncb890)

Blanchet MH, Le Good JA, Mesnard D, Oorschot V, Baflast S, Minchiotti G, Klumperman J \& Constam DB 2008 Cripto recruits Furin and PACE4 and controls Nodal trafficking during proteolytic maturation. EMBO Journal 27 2580-2591. (doi:10.1038/emboj.2008.174)

Brar AK, Handwerger S, Kessler CA \& Aronow BJ 2001 Gene induction and categorical reprogramming during in vitro human endometrial fibroblast decidualization. Physiological Genomics 7 135-148. (doi:10.1152/ physiolgenomics.00061.2001)

Brennan J, Norris DP \& Robertson EJ 2002 Nodal activity in the node governs left-right asymmetry. Genes and Development 16 2339-2344. (doi:10.1101/gad.1016202)

Carson DD, Bagchi I, Dey SK, Enders AC, Fazleabas AT, Lessey BA \& Yoshinaga K 2000 Embryo implantation. Developmental Biology 223 217-237. (doi:10.1006/dbio.2000.9767)

Chen Y \& Schier AF 2001 The zebrafish Nodal signal Squint functions as a morphogen. Nature 411 607-610. (doi:10.1038/35079121)

Chen Y \& Schier AF 2002 Lefty proteins are long-range inhibitors of squintmediated nodal signaling. Current Biology 12 2124-2128. (doi:10.1016/ S0960-9822(02)01362-3)
Chen C \& Shen MM 2004 Two modes by which Lefty proteins inhibit nodal signaling. Current Biology 14 618-624. (doi:10.1016/j.cub.2004.02.042)

Conlon FL, Barth KS \& Robertson EJ 1991 A novel retrovirally induced embryonic lethal mutation in the mouse: assessment of the developmental fate of embryonic stem cells homozygous for the 413.d proviral integration. Development 111 969-981.

Conlon FL, Lyons KM, Takaesu N, Barth KS, Kispert A, Herrmann B \& Robertson EJ 1994 A primary requirement for nodal in the formation and maintenance of the primitive streak in the mouse. Development $\mathbf{1 2 0}$ 1919-1928.

Cornet PB, Picquet C, Lemoine P, Osteen KG, Bruner-Tran KL, Tabibzadeh S, Courtoy PJ, Eeckhout Y, Marbaix E \& Henriet P 2002 Regulation and function of LEFTY-A/EBAF in the human endometrium. mRNA expression during the menstrual cycle, control by progesterone, and effect on matrix metalloprotineases. Journal of Biological Chemistry 277 42496-42504. (doi:10.1074/jbc.M201793200)

Cornet PB, Galant C, Eeckhout Y, Courtoy PJ, Marbaix E \& Henriet P 2005 Regulation of matrix metalloproteinase-9/gelatinase $\mathrm{B}$ expression and activation by ovarian steroids and LEFTY-A/endometrial bleeding-associated factor in the human endometrium. Journal of Clinical Endocrinology and Metabolism 90 1001-1011. (doi:10.1210/jc.2004-1277)

Dey SK, Lim H, Das SK, Reese J, Paria BC, Daikoku T \& Wang H 2004 Molecular cues to implantation. Endocrine Reviews 25 341-373. (doi:10.1210/er.2003-0020)

Gritsman K, Zhang J, Cheng S, Heckscher E, Talbot WS \& Schier AF 1999 The EGF-CFC protein one-eyed pinhead is essential for nodal signaling. Cell 97 121-132. (doi:10.1016/S0092-8674(00)80720-5)

Hamada H, Meno C, Watanabe D \& Saijoh Y 2002 Establishment of vertebrate left right asymmetry. Nature Reviews. Genetics 3 103-113. (doi:10.1038/nrg732)

lannaccone PM, Zhou X, Khokha M, Boucher D \& Kuehn MR 1992 Insertional mutation of a gene involved in growth regulation of the early mouse embryo. Developmental Dynamics 194 198-208. (doi:10.1002/ aja.1001940305)

Jones CM, Kuehn MR, Hogan BL, Smith JC \& Wright CV 1995 Nodalrelated signals induce axial mesoderm and dorsalize mesoderm during gastrulation. Development 121 3651-3662.

Jones RL, Salamonsen LA, Zhao YC, Ethier JF, Drummond AE \& Findlay JK 2002 Expression of activin receptors, follistatin and betaglycan by human endometrial stromal cells; consistent with a role for activins during decidualization. Molecular Human Reproduction 8 363-374. (doi:10.1093/molehr/8.4.363)

Kondo S 2002 The reaction-diffusion system: a mechanism for autonomous pattern formation in the animal skin. Genes to Cells 7 535-541. (doi:10.1046/j.1365-2443.2002.00543.x)

Kosaki K, Bassi MT, Kosaki R, Lewin M, Belmont J, Schauer G \& Casey B 1999 Characterization and mutation analysis of human LEFTY A and LEFTY B, homologues of murine genes implicated in left-right axis development. American Journal of Human Genetics 64 712-721. (doi:10.1086/302289)

Kothapalli R, Buyuksal I, Wu SQ, Chegini N \& Tabibzadeh S 1997 Detection of ebaf, a novel human gene of the transforming growth factor $\beta$ superfamily association of gene expression with endometrial bleeding. Journal of Clinical Investigation 99 2342-2350. (doi:10.1172/ JCI119415)

Kumar A, Novoselov V, Celeste AJ, Wolfman NM, ten Dijke P \& Kuehn MR 2001 Nodal signaling uses activin and transforming growth factor- $\beta$ receptor-regulated Smads. Journal of Biological Chemistry 276 656-661. (doi:10.1074/jbc.M004649200)

Liu G, Lin H, Zhang X, Li Q, Wang H, Qian D, Ni J \& Zhu C 2004 Expression of Smad2 and Smad4 in mouse uterus during the oestrous cycle and early pregnancy. Placenta 25 530-537. (doi:10.1016/j.placenta.2003.11.006)

Luo X, Xu J \& Chegini N 2003 The expression of Smads in human endometrium and regulation and induction in endometrial epithelial and stromal cells by transforming growth factor- $\beta$. Journal of Clinical Endocrinology and Metabolism 88 4967-4976. (doi:10.1210/jc.2003030276)

Ma GT, Soloveva V, Tzeng SJ, Lowe LA, Pfendler KC, lannaccone PM, Kuehn MR \& Linzer DI 2001 Nodal regulates trophoblast differentiation and placental development. Developmental Biology 236 124-135. (doi:10.1006/dbio.2001.0334) 
Meno C, Gritsman K, Ohishi S, Ohfuji Y, Heckscher E, Mochida K, Shimono A, Kondoh H, Talbot WS, Robertson EJ et al. 1999 Mouse Lefty2 and zebrafish antivin are feedback inhibitors of nodal signaling during vertebrate gastrulation. Molecular Cell 4 287-298. (doi:10.1016/ S1097-2765(00)80331-7)

Mesnard D \& Constam DB 2010 Imaging proprotein convertase activities and their regulation in the implanting mouse blastocyst. Journal of Cell Biology 191 129-139. (doi:10.1083/jcb.201005026)

Muller P, Rogers KW, Jordan BM, Lee JS, Robson D, Ramanathan S \& Schier AF 2012 Differential diffusivity of Nodal and Lefty underlies a reaction-diffusion patterning system. Science 336 721-724. (doi:10.1126/science.1221920)

Nadeem L, Munir S, Fu G, Dunk C, Baczyk D, Caniggia I, Lye S \& Peng C 2011 Nodal signals through activin receptor-like kinase 7 to inhibit trophoblast migration and invasion: implication in the pathogenesis of preeclampsia. American Journal of Pathology 178 1177-1189. (doi:10.1016/j.ajpath.2010.11.066)

Norris DP, Brennan J, Bikoff EK \& Robertson EJ 2002 The Foxh1-dependent autoregulatory enhancer controls the level of Nodal signals in the mouse embryo. Development 129 3455-3468.

Okada H, Nie G \& Salamonsen LA 2005 Requirement for proprotein convertase 5/6 during decidualization of human endometrial stromal cells in vitro. Journal of Clinical Endocrinology and Metabolism 90 1028-1034. (doi:10.1210/jc.2004-0904)

Papageorgiou I, Nicholls PK, Wang F, Lackmann M, Makanji Y, Salamonsen LA, Robertson DM \& Harrison CA 2009 Expression of nodal signalling components in cycling human endometrium and in endometrial cancer. Reproductive Biology and Endocrinology 7122. (doi:10.1186/1477-7827-7-122)

Park CB \& Dufort D 2011 a Nodal expression in the uterus of the mouse is regulated by the embryo and correlates with implantation. Biology of Reproduction 84 1103-1110. (doi:10.1095/biolreprod.110.087239)

Park CB \& Dufort D $2011 b$ Elsevier trophoblast research award lecture: the multifaceted role of Nodal signaling during mammalian reproduction. Placenta 32 (Suppl 2) 125-129. (doi:10.1016/j.placenta.2010.12.004)

Park CB, DeMayo FJ, Lydon JP \& Dufort D 2012 Nodal in the uterus is necessary for proper placental development and maintenance of pregnancy. Biology of Reproduction 86 194. (doi:10.1095/biolreprod.111.098277)

Pfendler KC, Yoon J, Taborn GU, Kuehn MR \& Iannaccone PM 2000 Nodal and bone morphogenetic protein 5 interact in murine mesoderm formation and implantation. Genesis 28 1-14. (doi:10.1002/1526968X(200009)28:1<1::AID-GENE10>3.0.CO;2-X)

Quaas A \& Dokras A 2008 Diagnosis and treatment of unexplained infertility. Reviews in Obstetrics and Gynecology 1 69-76.

Ramathal CY, Bagchi IC, Taylor RN \& Bagchi MK 2010 Endometrial decidualization: of mice and men. Seminars in Reproductive Medicine 28 17-26. (doi:10.1055/s-0029-1242989)

Rancourt SL \& Rancourt DE 1997 Murine subtilisin-like proteinase SPC6 is expressed during embryonic implantation, somitogenesis, and skeletal formation. Developmental Genetics 21 75-81. (doi:10.1002/(SICI)15206408(1997)21:1 < 75::AID-DVG9>3.0.CO;2-5)

Reissmann E, Jornvall H, Blokzijl A, Andersson O, Chang C, Minchiotti G, Persico MG, Ibanez CF \& Brivanlou AH 2001 The orphan receptor ALK7 and the Activin receptor ALK4 mediate signaling by Nodal proteins during vertebrate development. Genes and Development 15 2010-2022. (doi:10.1101/gad.201801)

Ross S \& Hill CS 2008 How the Smads regulate transcription. International Journal of Biochemistry and Cell Biology 40 383-408. (doi:10.1016/j. biocel.2007.09.006)

Sakuma R, Ohnishi Yi Y, Meno C, Fujii H, Juan H, Takeuchi J, Ogura T, Li E, Miyazono K \& Hamada H 2002 Inhibition of Nodal signalling by Lefty mediated through interaction with common receptors and efficient diffusion. Genes to Cells 7 401-412. (doi:10.1046/j.1365-2443.2002.00528.x)

Schier AF 2003 Nodal signaling in vertebrate development. Annual Review of Cell and Developmental Biology 19 589-621. (doi:10.1146/annurev. cellbio.19.041603.094522)

Schier AF 2009 Nodal morphogens. Cold Spring Harbor Perspectives in Biology 1 a003459. (doi:10.1101/cshperspect.a003459)

Stoikos CJ, Harrison CA, Salamonsen LA \& Dimitriadis E 2008 A distinct cohort of the TGF $\beta$ superfamily members expressed in human endometrium regulate decidualization. Human Reproduction 23 1447-1456. (doi:10.1093/humrep/den110)
Tabibzadeh S 2005 Role of EBAF/Lefty in implantation and uterine bleeding. In New Mechanisms for Tissue-Selective Estrogen-Free Contraception. Vol 52, pp 159-189. Eds HB Croxatto, R Schürmann, U Fuhrmann \& I Schellschmidt. Berlin, Germany: Springer Berlin Heidelberg. (doi:10.1007/3-540-27147-3_8)

Tabibzadeh S \& Hemmati-Brivanlou A 2006 Lefty at the crossroads of "stemness" and differentiative events. Stem Cells 24 1998-2006. (doi:10.1634/stemcells.2006-0075)

Tabibzadeh S, Kothapalli R \& Buyuksal I 1997 Distinct tumor specific expression of TGFB4 (ebaf)*, a novel human gene of the TGF- $\beta$ superfamily. Frontiers in Bioscience 2 a18-a25.

Tabibzadeh S, Lessey B \& Satyaswaroop PG 1998 Temporal and sitespecific expression of transforming growth factor- $\beta 4$ in human endometrium. Molecular Human Reproduction 4 595-602. (doi:10. 1093/molehr/4.6.595)

Tabibzadeh S, Mason JM, Shea W, Cai Y, Murray MJ \& Lessey B 2000 Dysregulated expression of ebaf, a novel molecular defect in the endometria of patients with infertility. Journal of Clinical Endocrinology and Metabolism 85 2526-2536. (doi:10.1210/jc.85.7.2526)

Takaoka K, Yamamoto M, Shiratori H, Meno C, Rossant J, Saijoh Y \& Hamada H 2006 The mouse embryo autonomously acquires anteriorposterior polarity at implantation. Developmental Cell 10 451-459. (doi:10.1016/j.devcel.2006.02.017)

Tang M, Xu Y, Julian J, Carson D \& Tabibzadeh S 2005a Lefty is expressed in mouse endometrium in estrous cycle and peri-implantation period. Human Reproduction 20 872-880. (doi:10.1093/humrep/deh717)

Tang M, Taylor HS \& Tabibzadeh S 2005 b In vivo gene transfer of lefty leads to implantation failure in mice. Human Reproduction 20 1772-1778. (doi:10.1093/humrep/deh849)

Tang M, Mikhailik A, Pauli I, Giudice LC, Fazelabas AT, Tulac S, Carson DD, Kaufman DG, Barbier C, Creemers JW et al. 2005C Decidual differentiation of stromal cells promotes proprotein convertase 5/6 expression and lefty processing. Endocrinology 146 5313-5320. (doi:10. 1210/en.2005-0684)

Tang M, Naidu D, Hearing P, Handwerger S \& Tabibzadeh S 2010 LEFTY, a member of the transforming growth factor- $\beta$ superfamily, inhibits uterine stromal cell differentiation: a novel autocrine role. Endocrinology 151 1320-1330. (doi:10.1210/en.2009-1081)

Torres PB, Florio P, Galleri L, Reis FM, Borges LE \& Petraglia F 2009 Activin A, activin receptor type II, nodal, and cripto mRNA are expressed by eutopic and ectopic endometrium in women with ovarian endometriosis. Reproductive Sciences 16 727-733. (doi:10.1177/1933719109334967)

Turing AM 1990 The chemical basis of morphogenesis. 1953. Bulletin of Mathematical Biology 52 153-197 (discussion 119-152). (doi:10.1007/ BF02459572)

Wang H \& Dey SK 2006 Roadmap to embryo implantation: clues from mouse models. Nature Reviews. Genetics 7 185-199. (doi:10.1038/nrg1808)

Wang H \& Tsang BK 2007 Nodal signalling and apoptosis. Reproduction 133 847-853. (doi:10.1530/REP-07-0053)

Wang H, Jiang JY, Zhu C, Peng C \& Tsang BK 2006 Role and regulation of nodal/activin receptor-like kinase 7 signaling pathway in the control of ovarian follicular atresia. Molecular Endocrinology 20 2469-2482. (doi:10.1210/me.2005-0446)

WHO Technical Report Series 1992 Recent advances in medically assisted conception. Report of a WHO Scientific Group. World Health Organization Technical Report Series 820 1-111.

Wood GA, Fata JE, Watson KL \& Khokha R 2007 Circulating hormones and estrous stage predict cellular and stromal remodeling in murine uterus. Reproduction 133 1035-1044. (doi:10.1530/REP-06-0302)

Zhou X, Sasaki H, Lowe L, Hogan BL \& Kuehn MR 1993 Nodal is a novel TGF- $\beta$-like gene expressed in the mouse node during gastrulation. Nature 361 543-547. (doi:10.1038/361543a0)

Received 22 March 2012

First decision 12 April 2012

Revised manuscript received 16 November 2012

Accepted 5 December 2012 